\title{
Different Features of Metabolic Connectivity Map and Granger Causality Method in Revealing Directed Dopamine Pathways: A Pilot Study Based on Integrated PET/MR
}

\section{Lei Wang}

Tangdu Hospital Fourth Military Medical University: Air Force Medical University Tangdu Hospital

\section{Longxiao Wei}

Tangdu Hospital Fourth Military Medical University: Air Force Medical University Tangdu Hospital

\section{Long Jin}

Tangdu Hospital Fourth Military Medical University: Air Force Medical University Tangdu Hospital

\section{Yunbo Li}

Tangdu Hospital Fourth Military Medical University: Air Force Medical University Tangdu Hospital

\section{Yixin Wei}

Tangdu Hospital Fourth Military Medical University: Air Force Medical University Tangdu Hospital Wei He

Tangdu Hospital Fourth Military Medical University: Air Force Medical University Tangdu Hospital

\section{Le Shi}

Tangdu Hospital Fourth Military Medical University: Air Force Medical University Tangdu Hospital

\section{Qinli Sun}

Xi'an Jiaotong University Medical College First Affiliated Hospital

\section{Wei Li}

Tangdu Hospital Fourth Military Medical University: Air Force Medical University Tangdu Hospital

\section{Qiang Li}

Tangdu Hospital Fourth Military Medical University: Air Force Medical University Tangdu Hospital

\section{Yongbin Li}

The first hospital of Xi'an

\section{Yarong Wang}

Xi'an Jiaotong University Medical College First Affiliated Hospital

\section{Menghui Yuan ( $\sim 13519196610 @ 139 . c o m$ )}

Tangdu Hospital Fourth Military Medical University: Air Force Medical University Tangdu Hospital

\section{Research Article}


Keywords: Metabolic Connectivity Map, Granger Causality, PET/MR, Reward Circuit, Dopamine

Posted Date: September 16th, 2021

DOI: https://doi.org/10.21203/rs.3.rs-842940/v1

License: (c) (1) This work is licensed under a Creative Commons Attribution 4.0 International License. Read Full License 


\section{Abstract}

Granger causality (GC) analysis and metabolic connectivity map (MCM) are two effective connectivity (EC) methods commonly used in functional brain researches. Although they have a common basis in central neurophysiology, their differences in depicting EC are not clear because of absenting data acquired simultaneously and exactly aligned. Integrated positron emission tomography and magnetic resonance image (PET/MR) technology makes this available. Using the "Monash rs-PET/MR" dataset obtained from the OpenNeuro database, we first conducted GC and MCM analysis of the brain dopamine reward circuit, a well-known system mainly consisting of the bilateral Orbital Frontal Cortex (OFC), Caudate (CAU), Nucleus Accumbens (NAc), Thalamus (THA) and Substantia Nigra (SN). Then, we validated their ability of describing EC to priori knowledge. The significance of each directed pathways within group were tested through one-sample t-test (for MCM) or Wilcoxcon test (for GC), the significance level was set at $p<0.05$ after FDR correction. Three types of connections were found: the cortico-nucleus (long-range), the nucleus-nucleus (neighborhood) and the symmetrical connections. GC revealed longrange connections including OFC-CAU and OFC-NAC; MCM revealed neighborhood connections including NAC-CAU, SN-THA, and THA-CAU, the symmetrical connections including the bilateral NAc, CAU, THA, as well as OFC-CAU. Thus, different patterns in directional networks of dopamine reward circuit revealed by GC and MCM. GC predominated at aspects of cortico-nucleus bidirected connections, while MCM of directed connections among close regions and symmetrical regions. This study implicates that research involving in effective connections should choose an appropriate analysis method according to the study purpose.

\section{Highlights}

- This is the first comparative study on effective connectivity methods based on integrated PET/MR data

- Different features were found of depicting directed dopamine pathways between metabolic connectivity map and granger causality

\section{Introduction}

The human brain has long been recognized as a continuously communicating network[1]. The multi-scale intrinsic activities, as small as neurons to brain regions, could be analyzed with functional connectivity (FC) to form the functional brain network. However, the networks built from correlation has no direction information. The effective connectivity (EC), which gives the directional or causal relationships among brain regions is more important for the exploration of the underlying brain mechanisms[2]. The understanding of the directionality of brain networks provides insights into the diagnosis and treatment of neurological or mental diseases such as Alzheimer's disease[3], schizophrenia[4] and addiction[5] etc.

Granger causality (GC) analysis is a commonly used approach in functional MRI studies to reveal the directionality between brain regions[6]. GC was originally introduced and operationalized by Wiener and 
Granger in economics[6], though linear vector autoregressive (VAR) model of stochastic time series data. The order of VAR model is usually determined by Bayesian information criterion (BIC) theory $[2,5,7]$. The basic concept of GC is that if the past of time series $X$ can be used to facilitate the prediction of the future of the time series $Y$, then we say there is a Granger causal influence from the $X$ to $Y$. GC method has been used broadly in neuroscience in the past decade, such as opioid addiction, migraine without aura, obsessive-compulsive disorder and $[5,8,9]$.

Metabolic connectivity map (MCM)[10] analysis is a novel approach to reveal the metabolic directionality among brain regions, using simultaneously collected positron emission tomography (PET) and functional magnetic resonance imaging (fMRI) data. This method is based on the concept of energy consumption in neuronal communication. Most energy metabolism is dedicated to signaling and is consumed up to $75 \%$ at the target neurons, post-synaptically [10][11-14]. Riedl et.al scaled this to the systems level, and assumed that an increase in local metabolism reflects an increase in afferent effective connectivity from source regions. They further hypothesized that the spatial correlations between metabolic activity and FC represents this EC spatial profile [10]. In other words, the MCM reflects the concordance between improved glutamate consumption pattern and the improved blood oxygen level dependent (BOLD) correlation pattern at the target region. As a novel and promising approach, MCM has been used in identifying the altered EC within default mode network for patients with Alzheimer's disease [3], in revealing the stable bidirectional connection between early/higher visual regions of healthy cohorts[10], and in studying the cognition task-induced reconfiguration of whole-brain networks[15].

The GC identifies the directional connectivity through the predictive relationship between BOLD signals while the MCM identifies this by the association between glucose metabolism and the FC. Since the level of glucose metabolism and the level of the BOLD signal are both influenced by the same cerebral blood flow, the two methods share a common basis in central neurophysiology. However, little has been known about the differences in depicting effective connectivity between GC and MCM due to the absenting fMRI and PET data acquired simultaneously and aligned exactly.

Dopamine (DA) plays several important roles in the human brain and has been researched for decades. The DA reward system has been well researched and described and is therefore suitable for the purpose of this comparative methodological study. Reward circuits mainly involves substantia nigra (SN), ventral tegmental area (VTA), thalamus (THA), ventral striatum, dorsal striatum and prefrontal cortex[16,17]. Three pathways consist the reward system: the nigrostriatal pathway, along which the $\mathrm{SN}$ sends a dopaminergic projection to the dorsal striatum; the meso-limbic pathway, where the VTA sends a similar type of dopaminergic projection to the NAc; the meso-cortical pathway, where the dopaminergic projections from SN/VTA through THA to prefrontal cortex[18,19]. Besides the dopaminergic projections, there are also GABAergic projections and glutamatergic projections among these DA areas, performing the inhibitory or excitatory functions[20].

With the development of integrated PET/MR technology, there is opportunity to compare GC and MCM using the same dataset. Although GC has a wider scale analysis ability from voxel-wise to regions-of- 
interest (ROI-wise), in contrast the MCM could only be applied to ROI-wise effective connectivity inference due to methodology issue currently. Thus, an ROI-wise effective connectivity analysis was used in this study. Based on the simultaneously collected PET and MRI data from a group of healthy participants, this exploratory study aims to reveal the sensitivity and consistency of the GC and MCM methods in identifying the directed pathways between brain regions. We assumed that both approaches could reveal important effective connectivity consistent with the DA pathway, but may not share the same pattern:

\section{Materials And Methods}

\subsection{Datasets}

The data of this study were obtained from the Monash rsPET-MR dataset[21-24] in the OpenNeuro database (https://doi.org/10.18112/openneuro.ds002898.v1.1.1). It is a simultaneous fMRI-fPET dataset acquired from young, healthy individuals at rest. Here is a brief description of this dataset: Participants ( $n=27,21$ female) were all right-handed, 18 to 23 years in age (mean 19 years), and 13 to 18 years of education (mean 14 years), and no history of diabetes, diagnosed Axis-1 mental illness or cardiovascular illness[21]. Participants underwent a 95-minute simultaneous MR-PET scan in a Siemens (Erlangen) 3 Tesla Biograph molecular MR scanner (Syngo VB20P). ${ }^{18}$ F-fluorodeoxyglucose (FDG; average dose 233MBq) was infused over the course of the scan[21]. Over the first 30-mins following infusion onset, T1 3D MPRAGE $\left(\mathrm{TA}=7.01 \mathrm{mins}, \mathrm{TR}=1640 \mathrm{~ms}, \mathrm{TE}=2.34 \mathrm{~ms}\right.$, flip angle $=8^{\circ}, \mathrm{FOV}=256 \times 256 \mathrm{~mm}^{2}$, voxel size $=1 \times 1 \times 1 \mathrm{~mm}^{3}, 176$ slices; sagittal acquisition); For the remainder of the scan, six consecutive 10 min blocks of T2 ${ }^{*}$-weighted echo-planar images (EPIs) were acquired $(\mathrm{TR}=2.45 \mathrm{~s}, \mathrm{TE}=30 \mathrm{~ms}, \mathrm{FOV}=$ $190 \mathrm{~mm}^{2}, 3 \times 3 \times 3 \mathrm{~mm}^{3}$ voxels, 44 slices, ascending axial acquisition)[21]. More details could be reviewed in previous paper[21,23].

\subsection{Image Preprocessing}

The first 10 min block BOLD data and the corresponding 10 min FDG PET data were used in this study, as well as the T1 3D MPRAGE data for structural segmentation and registration. According to the scan protocol[21], the PET data were reconstructed every 16 seconds to a volume, and the corresponding 10 min data should be volume number starting from 129 to 165 for each participant. These PET volumes were extracted and averaged to get the static FDG PET image.

CONN[25] toolbox (version 20.b) was used for BOLD data preprocessing. It is a functional connectivity toolbox based on the Statistical Parameter Mapping 12 toolbox (SPM, http:// www.fil.ion.ucl.ac.uk/spm). The default preprocessing pipeline for volume-based analyses (direct normalization to MNI-space) was utilized, including the following steps: Realignment and unwarp, slice timing correction for inter-slice differences in acquisition time, ART-based outlier detection (https://www.nitrc.org/projects/artifact_detect) to identify outlier scans for scrubbing, segmentation functional image and anatomical images to gray/white matter and cerebrospinal fluid (CSF) tissue classes using SPM unified segmentation and normalization procedure, and normalize to $2 \mathrm{~mm}$ 
(functional) or $1 \mathrm{~mm}$ (anatomical) isotropic voxel size in MNI space. Finally, the functional images were smoothed using spatial convolution with Gaussian kernel of $4 \mathrm{~mm}$ full width half maximum (FWHM). After preprocessing, functional data were further denoised using CONN's denoising pipeline, removing potential confounding effects in the BOLD signal such as noise components from white matter and CSF areas[26], estimated subject-motion parameters, scrubbing and session effects[27]. Temporal frequencies below $0.008 \mathrm{~Hz}$ or above $0.09 \mathrm{~Hz}$ are removed from the BOLD signal in order to focus on slow-frequency fluctuations while minimizing the influence of physiological, head-motion and other noise sources[28].

\subsection{ROI Definition and FC Analysis}

FSL[29] built-in atlases were used to generate 10 ROIs in dopamine system. Eight bilateral ROls were extracted from Harvard-Oxford probability atlas, thresholded at $50 \%$ probability, and then binarized into masks. These ROIs were thalamus (THA), nucleus accumbens (NAC), caudate (CAU) and orbital frontal cortex (OFC), bilaterally. Talairach Daemon atlas was used to generate bilateral substantia nigra (SN) ROI masks directly. For each participant, we calculated the ROI-wise and voxel-wise functional connectivity of every ROI, forming one FC matrix and $10 \mathrm{FC}$ maps per participant. The element value in FC matrices and voxel value in $\mathrm{FC}$ maps were further transformed to $\mathrm{z}$-value utilizing Fisher's $\mathrm{r}$ to $\mathrm{z}$ transformation equation, thus the distribution of z-value would approximately be normal. For ROI-wise FC matrices, one sample t-test was utilized to determine significant FC pathways within participant group. The significance level was defined by $p<0.05$, after false discover rate (FDR) multiple comparison correction.

\subsection{Granger Causality Analysis}

The granger causality indices $(\mathrm{GCl})$ of each ROI pairs were calculated along each significant FC pathways. This $\mathrm{GCl}$ was calculated using autoregressive model:

$$
\mathrm{Y}_{t}=\sum_{k=1}^{p} b_{k} Y_{(t-k)}+c Z_{t}+\varepsilon_{t}
$$

And the joint regressive representation was:

$$
\begin{gathered}
\mathrm{Y}_{t}=\sum_{k=1}^{p} a_{k} X_{(t-k)}+\sum_{k=1}^{p} b_{k} Y_{(t-k)}+c Z_{t}+\mu_{t} \\
F_{x \rightarrow y}=\ln \frac{\operatorname{var}\left(\varepsilon_{t}\right)}{\operatorname{var}\left(\mu_{t}\right)}
\end{gathered}
$$

Here, $Y_{t}$ and $X_{t}$ are the preprocessed ROI signals, $\varepsilon_{t}$ and $\mu_{t}$ represents the residuals of autoregression and joint regression, $\mathrm{p}$ means the lag of autoregression model. $F_{X \rightarrow y}$ is the $\mathrm{GCl}$ value defined as the granger causality effect from $R O I_{x}$ to $R O I_{y}$, so each $\mathrm{ROI}$ pair has two $\mathrm{GCl}$ value, representing $\mathrm{GC}$ effect of two directions. According to Bayesian information criterion[5], the lag of vector auto-regression model was determined to be 2 . 
While the distribution of $\mathrm{GCl}$ was not clear, the one sample t-test of group $\mathrm{GCl}$ statistical which needs the normal distribution assumption may not be appropriate. To get the real distribution of $\mathrm{GCl}$, permutationbased technology was adopted. We randomly switched ROI signals among participants preprocessed BOLD data, then calculated the GCl values of each ROI pairs, and repeat this procedure for 100,000 times. Finally, this generated a simulated $\mathrm{GCl}$ null distribution for real datasets. To test the significance of group $\mathrm{GCl}$, the non-parametric one-sample Wilcoxon signed rank test was utilized. If the $\mathrm{GCl}$ value of one directional connection was significantly higher than the median value of $\mathrm{GCl}$ null distribution, this directional connection should be considered significant ( $p<0.05$, FDR corrected).

\subsection{Metabolic Connectivity Map (MCM) Analysis}

Static FDG-PET images were preprocessed using FSL, including registration and normalization to $2 \mathrm{~mm}$ voxel size MNI space. Standard uptake value ratio (SUVr) maps were converted by dividing the mean value of reference region. According to previous research[30,31], cerebellum gray matter was chosen as the reference region. With regard to high-resolution PET data acquisition, there was less need to perform partial volume effect correction. The normalized SUVr maps and BOLD data of each participants were used to calculate MCM[10]. The MCM value of seed ROI $X$ to target ROI $Y$ was calculated as follows, the same method in the research of Riedl et.al $[3,10]$ :

$$
M C M_{X \rightarrow Y}=\operatorname{Correlation}\left(F C_{Y \mid X}, F D G_{Y}\right)
$$

In the formula, $F D G_{Y}$ is the voxel-wise profiles in $\mathrm{ROI} Y$, representing the neuronal activity in ROI $\mathrm{Y} ; F C_{Y I X}$ is the voxel-wise $\mathrm{FC}$ in $\mathrm{ROI} Y$ while $\mathrm{ROI} X$ as the seed ROI, representing the correlation between each voxel time series in ROI Y and the cluster time series of ROI X; The spatial correlation between FDG and FC voxel-wise profiles is the MCM value which represents the metabolic effective connectivity from ROI $X$ (seed) to ROI Y (target).

Based on the cellular model of neuro-energetics, a positive $M C M_{x \rightarrow y}$ value identifies the signaling input along the $\mathrm{FC}$ pathway from $\mathrm{ROI} X$ to ROI $\mathrm{Y}$. To identify the significant MCM connections among participant group, one-sample $t$-test were conducted for MCM values along each direction of FC pathways. The significance level was set at $p<0.05$, FDR corrected.

\section{Results}

\subsection{FC Analysis}

Among participants group, FC existed between most of the ROI pairs. The FC pathways connecting bilaterally symmetrical brain structures were significant for all ROIs, which were the FC between bilateral CAU, bilateral NAc, bilateral OFC, bilateral THA and bilateral SN. Besides, the FC between left SN and bilateral CAU, bilateral NAc, bilateral OFC, the FC between right SN and left CAU, bilateral NAc, left OFC, 
and the FC between right THA and bilateral OFC were not significant. All other FC between ROI pairs were significant except above mentioned pathways (Fig.1, One-sample $t$-test, $p<0.05$, FDR corrected).

\subsection{GC Analysis}

The distribution of $\mathrm{GCl}$ after 100,000 times simulation was shown in Fig.2. The $\mathrm{GCl}$ density curve was not normal but skewed, with a median value at 0.0163 . The mu parameter in Wilcoxon signed rank test thus was set as 0.0163 , and if the $\mathrm{GCl}$ was significantly higher than mu, the corresponding GC connection was considered significant. After multiple comparison correction, significant GC connections were as follows: a bidirectional connection between left NAc and right OFC and eight significant unidirectional connections respectively from right THA to left CAU, from left $\mathrm{CAU}$ to right $\mathrm{CAU}$, from left $\mathrm{CAU}$ to left $\mathrm{OFC}$, from left NAC to left $C A U$, from right OFC to left CAU, from left OFC to right CAU, from right NAc to left OFC, and from left OFC to right OFC (Fig.3, One-sample Wilcoxon signed rank test, $p<0.05$, FDR corrected).

\subsection{Analysis}

The significant MCM connections layover FC pathways were as follows: 3 bidirectional connections respectively between bilateral THA, between left CAU and left NAc, and between right CAU and right NAc, and 7 unidirectional connections respectively from left $S N$ to right THA, from right OFC to right CAU, from left OFC to bilateral CAU, from right NAc to bilateral CAU, and from left CAU to right CAU. (Fig.4, Onesample $t$-test, $p<0.05$, FDR corrected)

\section{Discussion}

In this present study, GC and MCM methods were applied to show differences in identifying the directional connectivity of brain reward system based on PET/MR dataset. Our findings demonstrated that GC was predominated at aspects of cortico-nucleus bidirected connections of reward system, while MCM of directed connections among adjacent regions and symmetrical region. Each of the two methods has its own features, and researchers should carefully choose them before starting analyzing.

Both GC and MCM identified some of these modulation pathways in DA systems, but with different patterns. According to our findings, GC did well in identifying long-range connections (cortico-nucleus). The bidirected connections between striatum to OFC were identified by GC, which is consistent with the meso-cortical pathway in reward circuits[32]. On the contrary, MCM did well in identifying directed connections among nearby brain areas (nucleus-nucleus) and among symmetrical brain areas. These tightly connections imply the frequent communications between dorsal and ventral striatum and also the intense communications between bilateral subcortical brain areas in resting state brain. The reason for these significant differences between GC and MCM may come from the deep nature of the two methods. GC's principal is based on temporal predictability of future activity from knowledge of past activity[33]. What GC revealed in the studies should be interpreted as predictions between time series[2]. If timeseries $\mathrm{X}$ and $\mathrm{Z}$ is highly correlated to each other (in other words, high FC between $\mathrm{X}$ and $\mathrm{Z}$ ), this high FC would 
cause lower GC between $X$ and $Z$, because GC uses lag to predict between timeseries, not correlation. According to GC's algorithm, an extreme example is that when $X$ and $Z$ is the same, there is obviously no $\mathrm{GC}$ effect at all. In our results, the FC between bilateral THA got highest value, but there was no significant GC pathway between bilateral THA. Besides, very high FC tends to get a lower GC, while this High-FC effect does not affect MCM. MCM's algorithm uses the voxel-wise spatial pattern similarity between FDG and FC to generate ROI-wise causality effect $[3,10]$. The nearby brain regions and symmetrical brain regions often have higher FC than long-distance brain regions. This may be the methodological reason that caused GC to give less results than MCM in nucleus-nucleus (nearby regions) pathways and symmetrical region pathways, as well as GC to give better results than MCM in cortico-nucleus (longrange) pathways. Although this inference is suggested by both the observed phenomenological and algorithmic differences, more stringent experiments are needed to verify this.

When the subtentorial areas were involved, MCM methods reported poor results and GC had even worse results. Only MCM identified the important projections from SN to THA, and the information interaction pathways between bilateral THA. The SN and THA were totally isolated from cortical and striatum according to our results, but the SN and THA should have interactions with cortical areas and striatum. This deficiency here may come from many factors. The first one could be the susceptibility of midbrain/brainstem areas to signal loss and image distortion for BOLD signals[34]. This reason explains why GC works even worse there. Due to GC's nature, it relies heavily on the BOLD signal quality. This disadvantage may have less effect for PET data. MCM got some benefit from high-resolution PET images than GC. Another factor may come from the lack of accuracy of the SN ROI template, considering the fact that $\mathrm{SN}$ is located in the mid-brain and is more likely to have poor registration results than cortex area using regular normalization procedure[34]. Besides, the correction method might be too stringent in our One-sample statistics when comparing to other studies which only focused on group differences on effective connectivity. For example, most of the GC studies calculated the between-group GC differences other than within-group patterns, so they could simply test whether the group differences were significantly not equal to zero. When we focused on within-group patterns of GC, we had to choose a more stringent value to test. This strategy may eventually cause less survived results. MCM, on the other hand, got less influence than GC when doing within-group analysis, and the results in subtenorial areas was better than GC.

This study has some limitations. Firstly, the reward circuit involved ROIs in this study was arbitrary selected and thus may cause the results less comprehensive. Secondly, the scan protocol of Riedl's study[10] was to acquire a 10min BOLD sequence right after the FDG injection and the PET data used in MCM analysis was collected 30 min after injection. While our dataset obtained from OpenNeuro acquires BOLD sequences 30 min after injection, which was not the same with Riedl's study[10]. It should be pointed out that in our study, the PET data represents the cumulated regional energy demands before the fMRI acquisition window, while the fMRI data reflects the neuronal dynamics during the acquisition phase. In theory, we could assume that the resting-state brain would have similar activities across sessions, thus the FC should not change a lot, which assures the MCM's stability. Whether MCM changes or not during resting-state in different sessions was not clear, thus further research is needed. Third, there 
are researches[1,35,36] providing improvements to original GC method, these variants claimed to be more reliable, but were not included in this study. These limitations remind us of the need for more cautious interpretation of results.

\section{Conclusion}

Both GC and MCM have their own features. Our results show that GC and MCM may reveal different patterns in directional networks. GC predominated at aspects of identifying long-range bidirected connections, while MCM of directed connections among adjacent regions and symmetrical regions. Poor connections in subtenorial areas were identified by both methods, but MEC got benefits from the compensation of the high-resolution PET data and worked better than GC. This implies that future research on effective connections requires an appropriate selection of methods to be conducted according to different objectives.

\section{Declarations}

\section{Funding:}

This work was supported by grants from the National Natural Science Foundation of China (82071497 to YR.W.).

\section{Conflicts of interest/Competing interests:}

The authors declare that they have no conflict of interest.

\section{Avaliability of data and material:}

Openneuro.org, ds002898, open accessed at https://doi.org/10.18112/openneuro.ds002898.v1.1.1

\section{Code availability:}

Custom code based on open source software. Questions regarding the code implemented in this work can be addressed to the corresponding author.

\section{Author's contributions:}

Lei Wang and Longxiao Wei contributed equally.

Menghui Yuan and Yarong Wang are the co-corresponding authors

\section{Ethics approval:}

Not applicable. All data from open accessed datasets from Openneuro.org. 
Not applicable.

\section{Consent for publications:}

Not applicable.

\section{Clinical Trial Registration:}

Not applicable.

\section{Acknowledgements:}

The authors are grateful to S.D. Jamadar for sharing such good dataset on OpenNeuro website, and professor Riedl Valentin's enthusiastic help with MCM methodology.

\section{References}

1. Wang X, Wang R, Li F, Lin Q, Zhao X, Hu Z. Large-Scale Granger Causal Brain Network based on Resting-State fMRI data. Neuroscience. 2020;425:169-80.

2. Cekic S, Grandjean D, Renaud O. Time, frequency, and time-varying Granger-causality measures in neuroscience. Stat Med. 2018;37:1910-31.

3. Scherr M, Utz L, Tahmasian M, Pasquini L, Grothe MJ, Rauschecker JP, et al. Effective connectivity in the default mode network is distinctively disrupted in Alzheimer's disease-A simultaneous restingstate FDG-PET/fMRI study. Hum Brain Mapp [Internet]. 2019 [cited 2019 Jun 16]; Available from: http://doi.wiley.com/10.1002/hbm.24517

4. Gao J, Zhang D, Wang L, Wang W, Fan Y, Tang M, et al. Altered Effective Connectivity in Schizophrenic Patients With Auditory Verbal Hallucinations: A Resting-State fMRI Study With Granger Causality Analysis. Front Psychiatry [Internet]. Frontiers; 2020 [cited 2021 Feb 5];11. Available from: https://www.frontiersin.org/articles/10.3389/fpsyt.2020.00575/full

5. Zhang Y, Li Q, Wen X, Cai W, Li G, Tian J, et al. Granger causality reveals a dominant role of memory circuit in chronic opioid dependence. Addict Biol. 2017. p. 1068-80.

6. Seth AK, Barrett AB, Barnett L. Granger Causality Analysis in Neuroscience and Neuroimaging. J Neurosci. Society for Neuroscience; 2015;35:3293-7.

7. Wen X, Liu Y, Yao L, Ding M. Top-Down Regulation of Default Mode Activity in Spatial Visual Attention. :10.

8. Huang X, Zhang D, Wang P, Mao C, Miao Z, Liu C, et al. Altered amygdala effective connectivity in migraine without aura: evidence from resting-state fMRI with Granger causality analysis. J Headache Pain. 2021;22:25.

9. Li H, Hu X, Gao Y, Cao L, Zhang L, Bu X, et al. Neural primacy of the dorsolateral prefrontal cortex in patients with obsessive-compulsive disorder. Neurolmage Clin. 2020;28:102432. 
10. Riedl V, Utz L, Castrillón G, Grimmer T, Rauschecker JP, Ploner M, et al. Metabolic connectivity mapping reveals effective connectivity in the resting human brain. Proc Natl Acad Sci. 2016;113:428-33.

11. Attwell D, Laughlin SB. An energy budget for signaling in the grey matter of the brain. J Cereb Blood Flow Metab Off J Int Soc Cereb Blood Flow Metab. 2001;21:1133-45.

12. Attwell $D$, ladecola $C$. The neural basis of functional brain imaging signals. Trends Neurosci. 2002;25:621-5.

13. Harris JJ, Jolivet R, Attwell D. Synaptic energy use and supply. Neuron. 2012;75:762-77.

14. Vergara RC, Jaramillo-Riveri S, Luarte A, Moënne-Loccoz C, Fuentes R, Couve A, et al. The Energy Homeostasis Principle: Neuronal Energy Regulation Drives Local Network Dynamics Generating Behavior. Front Comput Neurosci [Internet]. 2019 [cited 2021 Mar 25];13. Available from: https://www.ncbi.nlm.nih.gov/pmc/articles/PMC6664078/

15. Hahn A, Breakspear M, Rischka L, Wadsak W, Godbersen GM, Pichler V, et al. Reconfiguration of functional brain networks and metabolic cost converge during task performance. Yeo T, Behrens TE, Bijsterbosch JD, editors. eLife. eLife Sciences Publications, Ltd; 2020;9:e52443.

16. Ikemoto S. Brain reward circuitry beyond the mesolimbic dopamine system: A neurobiological theory. Neurosci Biobehav Rev. 2010;35:129-50.

17. Volkow ND, Wise RA, Baler R. The dopamine motive system: implications for drug and food addiction. Nat Rev Neurosci. 2017. p. 741-52.

18. Arias-Carrión O, Stamelou M, Murillo-Rodríguez E, Menéndez-González M, Pöppel E. Dopaminergic reward system: a short integrative review. Int Arch Med. BioMed Central; 2010;3:1-6.

19. Ikemoto S, Yang C, Tan A. Basal ganglia circuit loops, dopamine and motivation: A review and enquiry. Behav Brain Res. 2015;290:17-31.

20. Calabresi P, Picconi B, Tozzi A, Ghiglieri V, Filippo M. Direct and Indirect Pathways of Basal Ganglia: A Critical Reappraisal. Nat Neurosci. 2014;17:1022-30.

21. Jamadar SD, Ward PGD, Close TG, Fornito A, Premaratne M, O’Brien K, et al. Simultaneous BOLD$\mathrm{fMRI}$ and constant infusion FDG-PET data of the resting human brain. Sci Data. Nature Publishing Group; 2020;7:363.

22. Jamadar SD, Ward PGD, Carey A, Mclntyre R, Parkes L, Sasan D, et al. Radiotracer Administration for High Temporal Resolution Positron Emission Tomography of the Human Brain: Application to FDGfPET. JoVE J Vis Exp. 2019;e60259.

23. Jamadar SD, Ward PGD, Liang EX, Orchard ER, Chen Z, Egan GF. Metabolic and Hemodynamic Resting-State Connectivity of the Human Brain: A High-Temporal Resolution Simultaneous BOLDfMRI and FDG-fPET Multimodality Study. Cereb Cortex [Internet]. 2021 [cited 2021 Apr 21]; Available from: https://doi.org/10.1093/cercor/bhaa393

24. Jamadar SD, Ward PGD, Li S, Sforazzini F, Baran J, Chen Z, et al. Simultaneous task-based BOLDfMRI and [18-F] FDG functional PET for measurement of neuronal metabolism in the human visual cortex. Neurolmage. 2019;189:258-66. 
25. Whitfield-Gabrieli S, Nieto-Castanon A. Conn: a functional connectivity toolbox for correlated and anticorrelated brain networks. Brain Connect. 2012;2:125-41.

26. Behzadi Y, Restom K, Liau J, Liu TT. A component based noise correction method (CompCor) for BOLD and perfusion based fMRI. Neurolmage. 2007;37:90-101.

27. Power JD, Mitra A, Laumann TO, Snyder AZ, Schlaggar BL, Petersen SE. Methods to detect, characterize, and remove motion artifact in resting state fMRI. Neurolmage. 2014;84:320-41.

28. Hallquist MN, Hwang $K$, Luna $B$. The nuisance of nuisance regression: spectral misspecification in a common approach to resting-state fMRI preprocessing reintroduces noise and obscures functional connectivity. Neurolmage. 2013;82:208-25.

29. Jenkinson M, Beckmann CF, Behrens TEJ, Woolrich MW, Smith SM. FSL. Neurolmage. 2012;62:78290.

30. Shin J, Tsui W, Li Y, Lee S-Y, Kim SJ, Cho S-J, et al. Resting-State Glucose Metabolism Level Is Associated with the Regional Pattern of Amyloid Pathology in Alzheimer's Disease. Int J Alzheimers Dis [Internet]. 2011 [cited 2020 Jan 3];2011. Available from: https://www.ncbi.nlm.nih.gov/pmc/articles/PMC3065040/

31. Wang J, Shan Y, Dai J, Cui B, Shang K, Yang H, et al. Altered coupling between resting-state glucose metabolism and functional activity in epilepsy. Ann Clin Transl Neurol. 2020;7:1831-42.

32. Volkow ND, Michaelides M, Baler R. The Neuroscience of Drug Reward and Addiction. Physiol Rev. 2019;99:2115-40.

33. Chen H, Uddin LQ, Zhang Y, Duan X, Chen H. Atypical effective connectivity of thalamo-cortical circuits in autism spectrum disorder: Impaired thalamo-cortical connectivity in ASD. Autism Res. 2016;9:1183-90.

34. de la Cruz F, Wagner G, Schumann A, Suttkus S, Güllmar D, Reichenbach JR, et al. Interrelations between dopamine and serotonin producing sites and regions of the default mode network. Hum Brain Mapp. 2020;42:811-23.

35. Seth AK, B.Barrett A, Barnett L. Granger Causality Analysis in Neuroscience and Neuroimaging. 2016.

36. Luo Q, Lu W, Cheng W, Valdes-Sosa PA, Wen X, Ding M, et al. Spatio-temporal Granger causality: A new framework. Neurolmage. 2013;79:241-63.

\section{Figures}




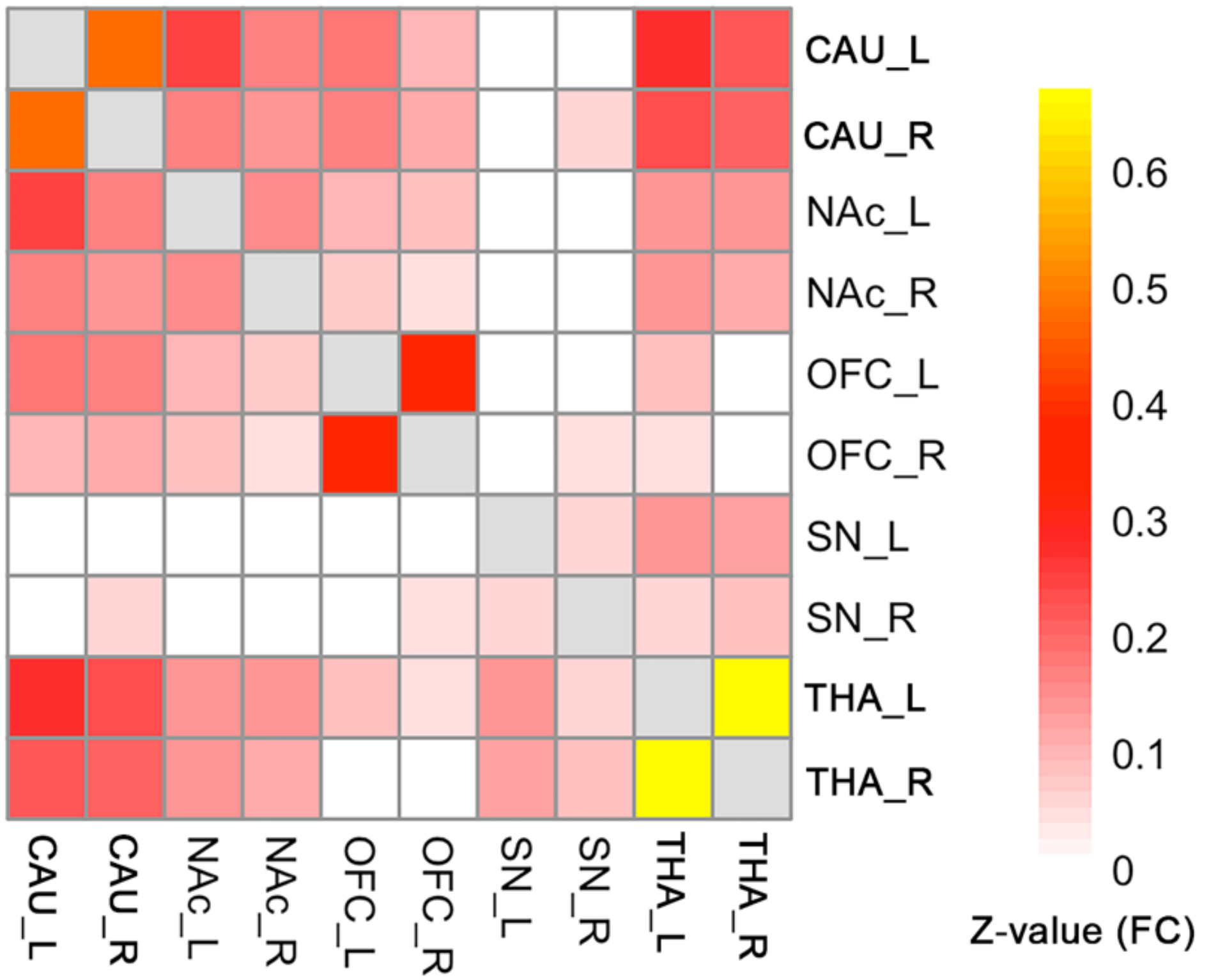

Figure 1

The Group-level FC matrix shows that the bilaterally CAU, NAc, OFC and THA ROIs were significantly connected to each other. The SN and were significantly connected to THA. Color represents the ztransformed FC strength between ROI pairs. White color means statistically not significant. (One-sample ttest, p<0.05, FDR corrected) CAU: Caudate, NAc: Nucleus Accumbens, OFC: Orbital Frontal Cortex, SN: Substantia Nigra, THA: Thalamus 


\section{GCI density curve}

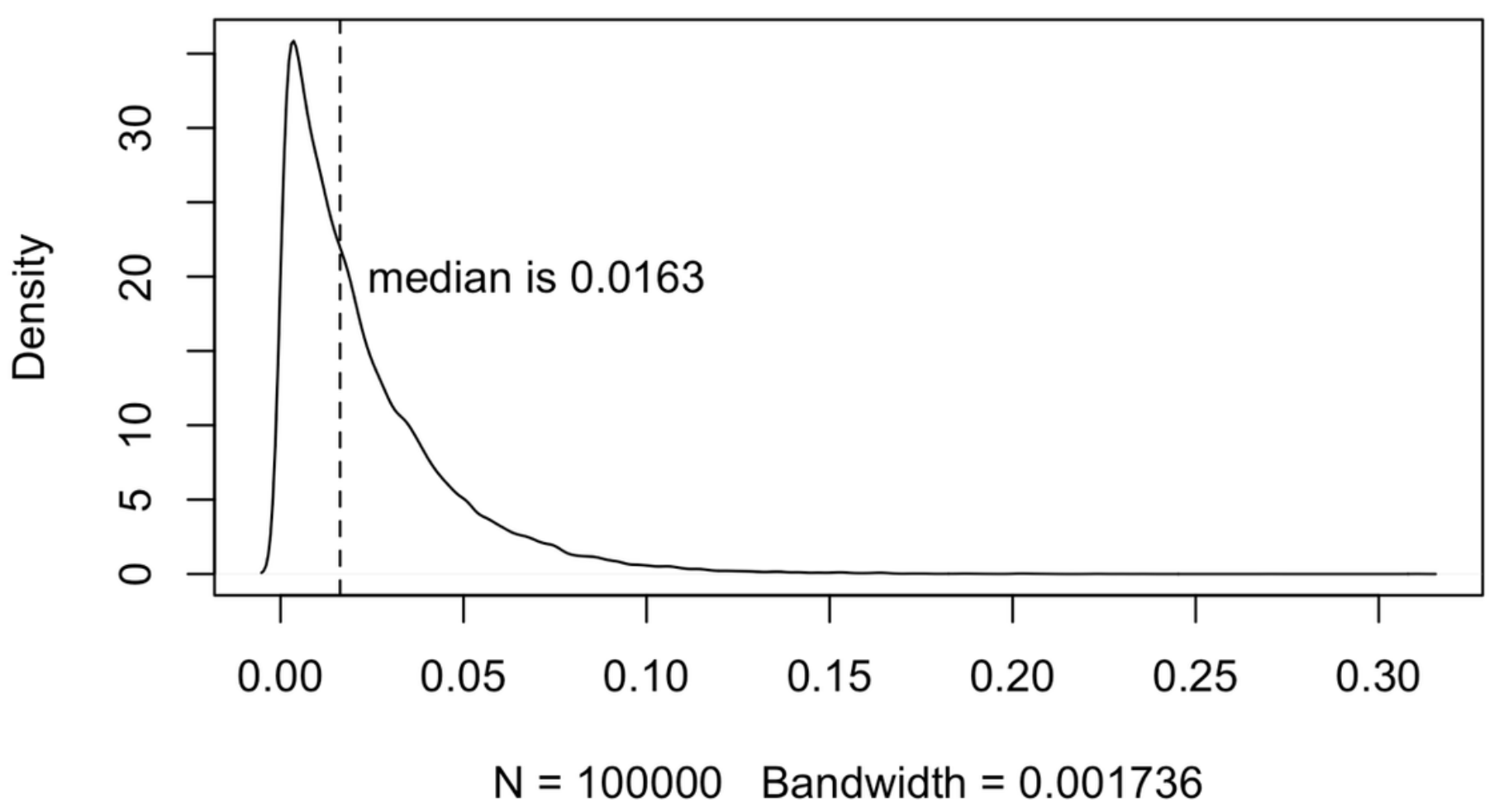

Figure 2

The GCl density curve. This curve comes from permutation-based technology to simulates real data $\mathrm{GCl}$ distribution with 100,000 times permutation. The distribution was a skewed curve, with 0.0163 as the median value, which is more appropriate than mean value for one-sample test in this situation. 

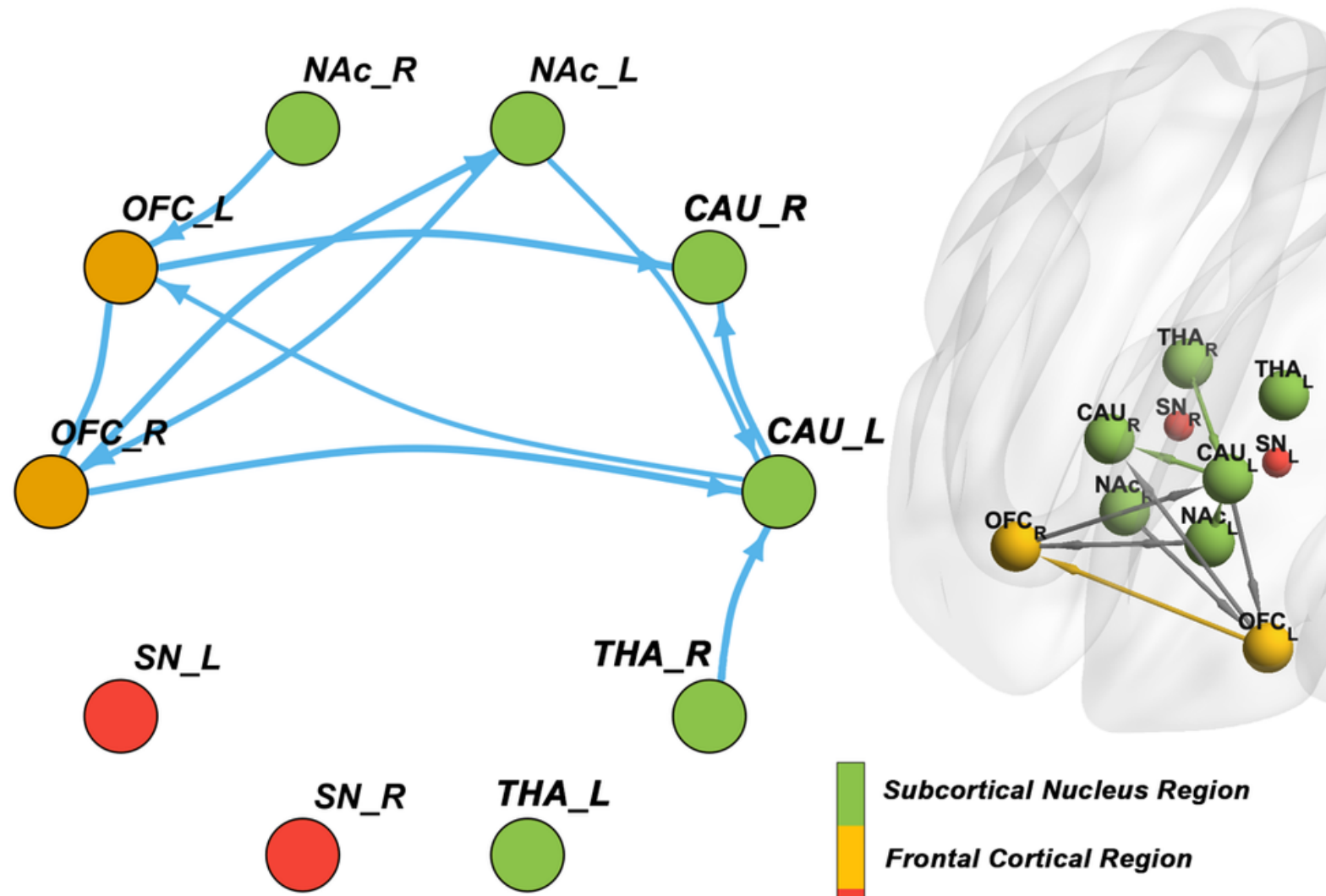

Subcortical Nucleus Region

Frontal Cortical Region

Brainstem/Midbrain Region

Figure 3

The group-wise directional network identified by Granger causality. Arrows represent the direction of the pathways. The line thickness represents the relative strength of the granger causality index of that pathway. Bidirectional connection was identified between the right OFC and left NAc. (One-sample Wilcoxon signed rank test, $\mathrm{p}<0.05$, FDR corrected) CAU: Caudate, NAc: Nucleus Accumbens, OFC: Orbital Frontal Cortex, SN: Substantia Nigra, THA: Thalamus, L/R: Left / Right 

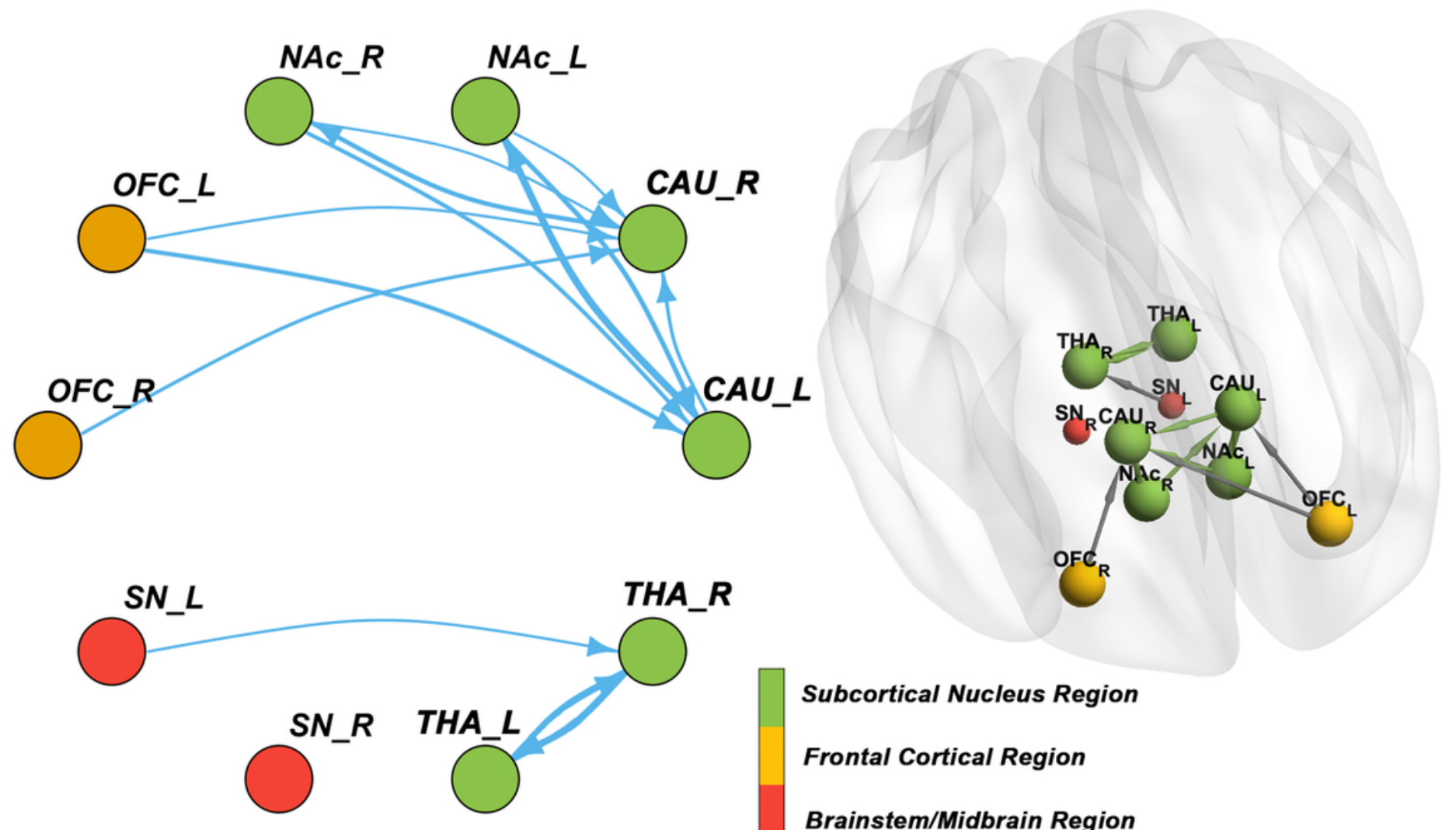

Subcortical Nucleus Region

Frontal Cortical Region

Brainstem/Midbrain Region

\section{Figure 4}

The group-wise directional pathways identified by Metabolic effective connection (MEC) method. Arrows represent the direction of the pathways. The line thickness represents the relative strength of the MEC. There were more bidirectional interactions between NAc and caudate, and the unidirectional pathway from OFC to caudate revealed the regulation in frontostriatal dopamine pathway. (One-sample t-test, p<0.05, FDR correted) CAU: Caudate, NAc: Nucleus Accumbens, OFC: Orbital Frontal Cortex, SN: Substantia Nigra, THA: Thalamus, L/R: Left / Right 